\title{
Wisp2 disruption represses Cxcr4 expression and inhibits BMSCs homing to injured liver
}

\author{
Dan Qin ${ }^{1, *}$, Yi Yan ${ }^{1, *}$, Bian Hu ${ }^{2, *}$, Wanpo Zhang ${ }^{1}$, Hanmin Li ${ }^{3}$, Xiaodong $\mathrm{Li}^{3}$, Shenghui \\ Liu ${ }^{1}$, Depeng Dai ${ }^{1}$, Xiongji Hu${ }^{1}$, Xingxu Huang ${ }^{2}$ and Lisheng Zhang ${ }^{1}$ \\ ${ }^{1}$ College of Veterinary Medicine, University of Huazhong Agricultural, Wuhan 430070, People's Republic of China \\ ${ }^{2}$ School of Life Science and Technology, Shanghai Tech University, Pudong New Area, Shanghai 201210, People's Republic \\ of China \\ ${ }^{3}$ Hepatic Disease Institute, Hubei Provincial Hospital of Traditional Chinese Medicine, Wuhan 430061, People's Republic of \\ China \\ *These authors have contributed equally to this work
}

Correspondence to: Lisheng Zhang, email: lishengzhang@mail.hzau.edu.cn

Keywords: rat; Wisp2; Cxcr4; BMSCs; homing

Received: June 10,2017 Accepted: October 02, $2017 \quad$ Published: October 24, 2017

Copyright: Qin et al. This is an open-access article distributed under the terms of the Creative Commons Attribution License 3.0 (CC BY 3.0), which permits unrestricted use, distribution, and reproduction in any medium, provided the original author and source are credited.

\section{ABSTRACT}

Liver regeneration/repair is a compensatory regrowth following acute liver failure, and bone marrow-derived mesenchyme stem cell (BMSC) transplantation is an effective therapy that promotes liver regeneration/repair. Wnt1 inducible signaling pathway protein 2 (Wisp2) is highly expressed in BMSCs, however, its function remains unclear. In this work, we used clustered regularly interspaced short palindromic repeats (CRISPR)-associated protein -9 nuclease (CRISPR/Cas9) genome editing technology to knockdown Wisp2 in BMSCs, and these modified cells were then transplanted into rats which were induced by the 2-AAF/PH. By linking the expression of Cas9 to green fluorescent protein (GFP), we tracked BMSCs in the rats. Disruption of Wisp2 inhibited the homing of BMSCs to injured liver and aggravated liver damage as indicated by remarkably high levels of ALT and AST. Moreover, the key factor in BMSC transplantation, C-X-C chemokine receptor type 4 (Cxcr4), was down-regulated in the Wisp2 depleted BMSCs and had a lower expression in the livers of the corresponding rats. By tracing the GFP marker, more BMSCs were observed to differentiate into CD31 positive endothelial cells in the functional Wisp2 cells but less in the Wisp2 gene disrupted cells. In summary, Wisp2 promotes the homing of BMSCs through Cxcr4 related signaling during liver repair in rats.

\section{INTRODUCTION}

Liver cancer is one of the most frequently diagnosed cancers worldwide and one of the leading causes of cancer death. An estimated 782,500 new liver cancer cases and 745,500 deaths occurred worldwide in 2012. Half of these cases and deaths were estimated to occur in China $[1,2]$. Acute liver injury (ALI) and acute liver failure (ALF) are syndromes characterized by a rapid loss of functional hepatocytes in patients with no evidence of pre-existing liver disease. Despite aggressive medical management, many patients with ALF deteriorate severely, and liver transplantation remains their only option for survival [3]. Because of the limited donor availability, attention has been focused on the possibility of restoring liver mass and function through cell transplantation [4]. Recently, studies have documented that bone marrow cells can migrate to injured liver and differentiate into local cell types in the absence of resident cell proliferation. Thus, bone marrow cells can be considered a pool from which exogenous cells 
could be derived for liver regeneration/repair [5], and among those cells, bone marrow-derived mesenchyme stem cells (BMSCs) have the most potential [6].

BMSCs can be cultivated from bone marrow aspirates as plastic adherent cells in vitro [7]. It is a type of stem cell with powerful proliferative and differential potential that represent an attractive tool for the establishment of successful stem cell-based therapy for liver diseases. A number of different stromal cells contribute to the therapeutic effects exerted by BMSCs because BMSCs can differentiate into functional hepatic cells and produce a series of growth factors and cytokines capable of suppressing inflammatory responses, reducing hepatocyte apoptosis, reversing liver fibrosis and enhancing hepatocyte functionality [8]. Besides, BMSCs have an inherent capacity to home to an injured liver and enhance wound healing [9]. BMSCs are safer than embryonic stem cells to use in vivo due to their higher chromosomal stability and lower tendency to form neoplasms in the recipient host [10]. However, many researchers found that these cells are poorly efficient in cell transplantation $[11,12]$. Retrospective studies have revealed that the low colonization of transplanted MSCs in the liver was the main factor restricting the efficacy of MSC transplantation [13, 14]. To date, an increasing number of factors have been shown to participate in MSC homing, including cytokines, chemokines, growth factors, bioactive lipids, and adhesion molecules [9]. Despite this general understanding of MSC homing, much work is needed to elucidate the molecular mechanisms responsible for this process.

Wnt1 inducible signaling pathway protein 2 (Wisp2, also known as CCN5) is a member of the connective tissue growth factor/cysteine- rich 61/ nephroblastoma overexpressed (CCN) family $[15,16]$. It is a $29-\mathrm{kDa}$ protein that is secreted by adipose precursor cells and fibroblasts and is highly expressed in MSCs [17-19]. Early discoveries have led to further research regarding its roles in cell signaling, proliferation, adhesion, invasion, wound healing, fibrosis, skeletal development, implantation, epithelial-mesenchyme transition, angiogenesis and cancers [20-23]. Wisp2 has both growth-promoting and growth-arresting properties depending on the cell types and the microenvironment of the cells [24]. Wisp2 mRNA and protein were overexpressed in preneoplastic and cancerous cells in human breast. Moreover, the disruption of Wisp2 signaling in MCF-7 cells using antisense oligomers caused a significant reduction in tumor cell proliferation [25]. However, the loss of Wisp2, which is observed in colorectal cancer (CRC), may lead to increased tumor cell invasion and disease progression. Wisp2 may, thus, has potential for therapeutic strategies for the treatment of CRC metastasis [20]. In addition, secreted Wisp2 is a novel regulator of canonical Wnt activation and maintains MSCs in an undifferentiated state. Simultaneously, Wisp2 inhibited Ppary and associated adipose genes and, similarly to WNT3a, promoted the partial dedifferentiation of the cells and the induction of a myofibroblast phenotype, leading to the activation of markers of fibrosis [17]. Recently, researchers found that Wisp2 might be an important regulator of BMSCs in the regulation of proliferation and initiation of specific differentiation pathways [18, 24], but little is known regarding its mechanism.

To better understand the mechanism underlying the impact of Wisp2 in vitro and in vivo, we used clustered regularly interspaced short palindromic repeats (CRISPR)associated protein-9 nuclease (CRISPR/Cas9) gene editing technology [21] to knockdown Wisp2 expression. One of the most striking findings of this analysis was an apparent down-regulation of $\mathrm{C}-\mathrm{X}-\mathrm{C}$ motif receptor 4 (Cxcr4) both in vitro and in vivo, followed by the loss of Wisp2. Furthermore, by tracking GFP-labeled BMSCs, we found that the loss of Wisp2 resulted in reduced homing and a deterioration of liver function. Notably, CD31, which is the endothelial cell specific marker, was significantly suppressed in the Wisp2 disrupted BMSC transplanted rats. These results illustrate that Wisp2 controls the homing of BMSCs that maybe related to Cxcr4 signaling during liver repair and thus provide a therapeutic roadmap for achieving hepatic regeneration and repair.

\section{RESULTS}

\section{High expression of Wisp2 in BMSCs}

Wisp2 has been reported to be expressed in BMSCs, to explore the function of Wisp2, we detected its expression by PCR and western blot. As shown in Figure 1a, $1 \mathrm{~b}$ and 1c, Wisp2 was highly expressed in BMSCs but lowly expressed in rat liver.

\section{Establishment of Wisp2 knockdown cells by CRISPR/Cas9 system}

To further investigate the role of Wisp2 in the process of liver repair, the CRISPR/Cas9 system was adopted and eight sgRNAs were designed and worked in pairs (Table 1). The efficiencies of all sgRNAs were first tested by cloning into the Px330 vector and then transfecting into NIH3T3 fibroblasts. It is shown that different sgRNAs had different cutting efficiency. Notably, sgW(5+6) and sgW(7+8) had higher specificity (Supplementary Figure 1a). Next, we cloned these two pairs into a lentivirus vector (Figure 2a) and verified their cleavage efficiency by transducing into Hep16 cells. The T7EN1 cleavage bands were visible in the targeted genes (Supplementary Figure 1b and 1c). These data demonstrated that the designed sgRNAs worked effectively with Cas9 in the targeted genes in the cultured cells. 
To obtain stable knockdown cell lines, a lentivirus plasmid containing $\operatorname{sgW}(7+8)$ was co-transfection with pMD2.G and PsPAX2 in 293T cell lines for lentivirus generation. Then, the $\mathrm{C} 3 \mathrm{H} 10 \mathrm{~T} 1 / 2$ cells, which are murine mesenchymal stem cells, were infected with lentivirus targeting Wisp2 or control virus. For further verification, a T7EN1 cleavage assay was performed, and the cleavage bands were visible in the Wisp2-sgRNA group (Figure $2 b$ and $2 \mathrm{c}$ ). In addition, the cleavage was characterized by Sanger sequencing, which displayed overlapped peaks in the sequencing chromatographs (Figure 2d). Furthermore, the mRNA expression of Wisp 2 was determined by qRTPCR. As shown in Figure 2e, Wisp2 expression was significantly decreased in the Wisp2-sgRNA group.

Wisp2 is reported to be correlated positively with Cyclin D1 and inhibits Ppary activation. Our data showed that disruption of Wisp2 significantly decreased Cyclin D1 abundance (Figure 2f) and increased Ppary abundance (Figure 2g).

Altogether, these results showed that the selected sgRNAs worked effectively with Cas9 and the CRISPR / Cas9 system functions well in vitro.

\section{The expression of Cxcr4 was reduced in the Wisp2 genetically modified cells}

Based on the experiment above, BMSCs were infected with CRISPR/Cas9 lentivirus targeting the Wisp2 gene or control virus, and Supplementary Figure 2a represented high-efficiency retroviral infection of BMSCs. Subsequently, genomic DNA was extracted and PCR and T7EN1 digestion were performed, the T7EN1 cleavage bands were visible in the targeted gene (Figure $3 \mathrm{a}$ and $3 b)$. At the molecular level, the cleavage was characterized further by Sanger sequencing, which displayed overlapped peaks in the sequencing chromatographs (Supplementary Figure 2b). Next, a routine determination was performed via qRT-PCR to detect Wisp2 expression. Unsurprisingly, the mRNA level of Wisp2 was significantly reduced (Figure 3c).

According to previous studies, Cxcr4 stimulates the migration of MSCs in vitro and is known to be required for the migration of MSCs in vivo, particularly in the liver. Thus, the expression of Cxcr4 in the Wisp2-sgRNA cells was analyzed, and the abundance of Cxcr4 was shown to be dramatically decreased (Figure 3d). Then, Cxcr4 was examined by immunoblotting and immunostaining. As shown in Figure 3e and Figure 3f, Cxcr4 was markedly reduced in the Wisp2-sgRNA BMSCs. The same phenomenon was also detected in the C3H10T1/2 cells at the mRNA level (Supplementary Figure 2c). Besides, the mRNA expression of $\mathrm{Cxcr} 7$ was reduced, concomitant with the decreased Cxcr 4 in both modified cell lines (Supplementary Figure 2d and 2e).

Altogether, the loss of Wisp2 significantly reduced the expression of Cxcr 4 in the cultured cells from both mice and rats.

\section{Impaired liver repair in the lentiviral Wisp2 disrupted BMSC transplantation rats after 2-AAF/PH induced liver injury}

To further determine the contribution of Wisp2 to BMSC-mediated liver regeneration and repair, the Wisp2 modified BMSCs were transplanted into injured livers of rats subjected to 2-AAF/PH (Figure 4a). By tracing GFP, BMSCs were detected in rat liver at day 2 and day 10 in Figure $4 \mathrm{~b}$. Compared to the control group, the expression of Wisp2 was significantly decreased in the Wisp2-sgRNA group (Figure 4c and 4d).

Our preceding results indicated that the loss of Wisp2 disrupted the expression of Cxcr4 from both mice and rat MSCs, we, therefore, investigated whether Wisp2 played the same role in this liver repair model. As expected, Cxcr4 was reduced in the Wisp2 deletion group compared to that in the functional Wisp2 BMSC a

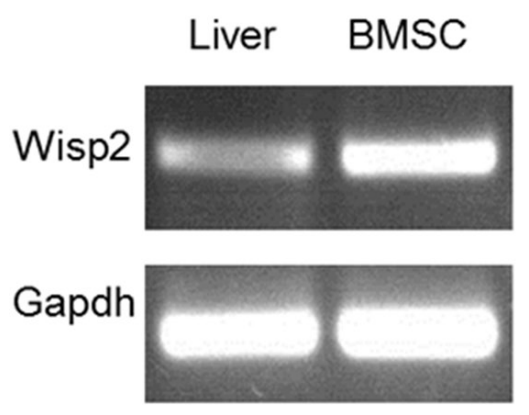

b

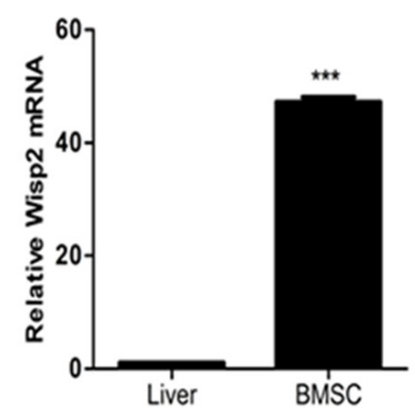

C

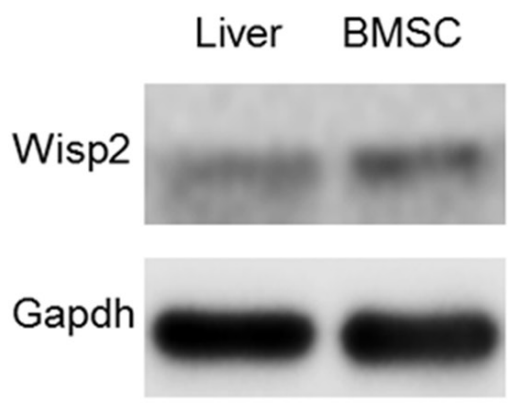

Figure 1: High expression of Wisp2 in BMSCs. Total RNA was extracted from rat BMSCs or rat livers, then PCR (a) and qRT-PCR (b) were performed to examine Wisp2 mRNA level. Error bars represent the \pm S.E.M. of triplications. “***”, p $<0.001$. Gene expression level of the samples normalized to Gapdh and the expression of Wisp2 in liver was set as 1. (c) The protein level of Wisp2 was measured in BMSCs and livers with western blot analysis. $20 \mu \mathrm{g}$ total protein per lane and Gapdh was used as an internal control. 
Table 1: Sequences of sgRNAs

\begin{tabular}{ll}
\hline sgRNAs & Sequences \\
\hline Wisp2 sgRNA1 & GGGTACCCCTGGTGCTGGA \\
Wisp2 sgRNA2 & GTGTCCAAGGACAGGCACA \\
Wisp2 sgRNA3 & GGCCTGGTTTGTCAGCCTG \\
Wisp2 sgRNA4 & CAGACATGCAGGTGGTCGC \\
Wisp2 sgRNA5 & GTGAATGGCCGCAGGTACC \\
Wisp2 sgRNA6 & TCATCCTCTTCGACTGCAC \\
Wisp2 sgRNA7 & GAGAATACAGGTGCCAGGA \\
Wisp2 sgRNA8 & GCACATCCTCACTGCACAG \\
\hline
\end{tabular}

transplanted group (Figure 5a). Additionally, the proinflammation factor, interleukin-6 (IL-6) was increased in the Wisp2 disrupted group (Figure 5b).

To investigate the liver histology of the rats after transplantation of the BMSCs, hematoxylin and eosin (H\&E) staining was performed. Compared to the control group, the livers in the Wisp2-sgRNA group exhibited cell shrinkage with a disappeared cytoplasm and vacuolar degeneration (Figure 5c). Additionally, some of the hepatocytes dissolved, allowing the cells to develop soluble necrosis. Furthermore, some of these cells disintegrated directly, and cellular debris could be observed (Supplementary Figure 3a). Proliferating cell nuclear antigen (PCNA) and $\alpha$-smooth muscle actin ( $\alpha$-SMA) staining were based on H\&E staining to detect the proliferation and vascular fibrosis. PCNA staining revealed that growth efficiency was much lower in Wisp2 gene disruption group, the result of $\alpha$-SMA staining was exactly the opposite (Figure 5c).

Since ALT and AST are commonly measured clinically as a part of the diagnostic evaluation of hepatocellular injury, we compared the serum ALT/AST in the control rats and the Wisp2-sgRNA BMSC transplanted rats. Predictably, the serum AST and ALT levels were significantly higher in the Wisp2 depleted rats (Figure 5d and 5e). Similarly, terminal deoxynucleotidyl transferase 2-deoxyuridine, 5-triphosphate nick end labeling (TUNEL) staining revealed that disrupted Wisp2 induced apoptosis compared with the control group (Figure 5f). Thus, we demonstrated that loss of Wisp2 prevented the damaged liver from repairing.

\section{Deletion of Wisp2 reduced the in vivo homing of transplanted BMSCs to the injured livers related to Cxcr4 signaling}

To elucidate the mechanism underlying the proregeneration and repair of BMSCs niche, gene expression was detected by immunofluorescence staining. The expression of Wisp2 was conditionally ablated in the Wisp2-sgRNA group (Figure 6a). Figure $6 \mathrm{~b}$ indicated that Cxcr4 was reduced in the Wisp2 deletion group compared to that in the functional Wisp2 BMSC transplanted group. In addition, by tracing GFP, we found that the homing efficiency of the functional Wisp2 BMSCs in the transplanted rats was much higher than that in the Wisp2 modified rats (Figure 6a-6c). Meanwhile, the transplanted BMSCs spread from the blood vessels along the hepatic sinus to the periphery (Supplementary Figure $3 b$ ).

To determine how BMSCs modulated liver regeneration and repair, specific markers were detected, including CD31, cytokeratin 19(CK19) and von willebrand factor(vWF). Finally, we discovered that CD31 was much more highly expressed in control group than in the Wisp2-sgRNA group (Figure 6c). Compared to CD31, CK19 staining displayed less co-expression and more self-signal (Supplementary Figure 4a), in contrast, hardly positive vWF co-dyeing signal could be observed (Supplementary Figure 4b).

Hence, it was possible that BMSCs repair liver injury by endothelial cell proliferation and angiogenesis through Wisp2 mediated Cxcr4 signaling.

\section{DISCUSSION}

Recently, the possibility of using organ- or tissuespecific adult stem cells has been examined in both fields of gene and cell transplantation therapy [5]. One of the main attractions was MSCs, particularly for the treatment of ALF [13, 22]. Unfortunately, many researchers have found that cell engraftments have a poor efficacy [23]. This report showed that Wisp2 knockdown by CRISPR/ Cas9 system inhibited BMSCs homing to injured livers through $\mathrm{Cxcr} 4$ related signaling and delayed liver repair.

MSCs exhibit multiple beneficial properties due to their capacity for homing, attenuating the inflammatory response, modulating immune cells, and promoting tissue healing [26]. The great benefit of BMSCs is that they are easily accessible through aspiration of the patient's bone marrow, so that the use of BMSCs avoids ethical issues, facilitating their application both for auto- and allo-transplantation. BMSCs are also easily expanded on a large scale, which is very convenient for clinical use [27]. Here, we transplanted BMSCs labeled with GFP via the 


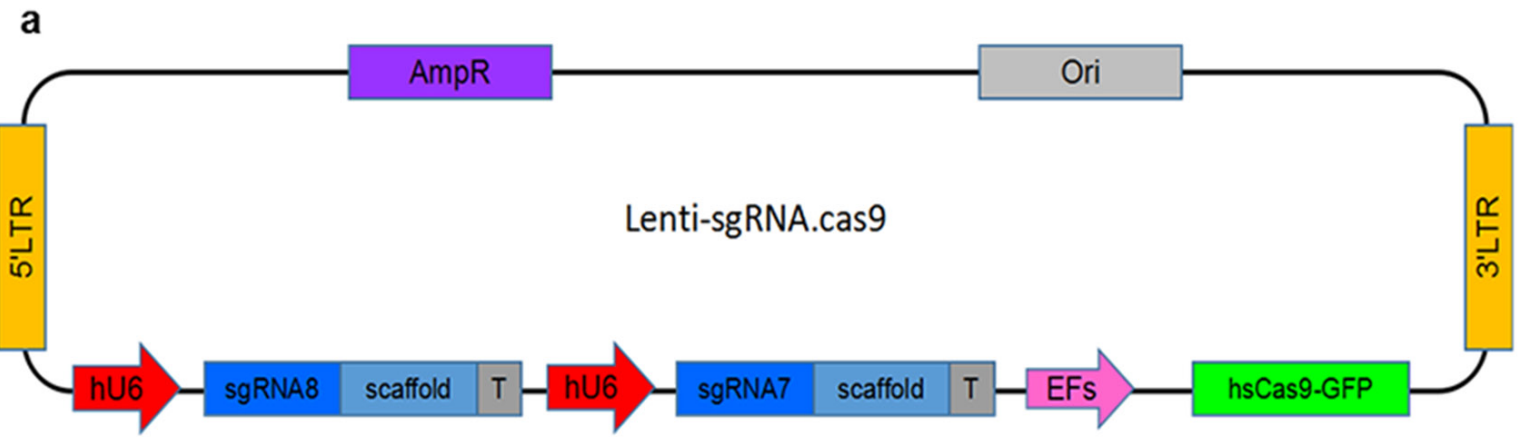

b
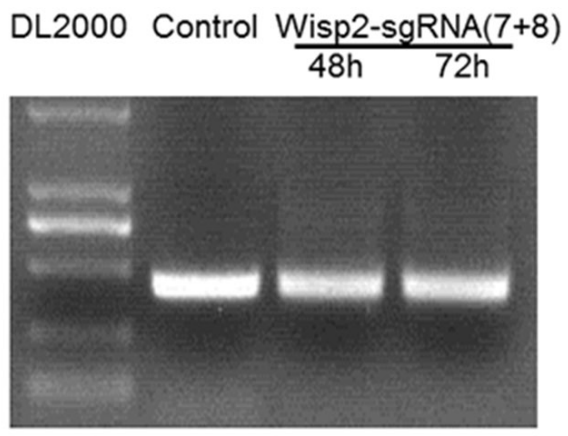

C

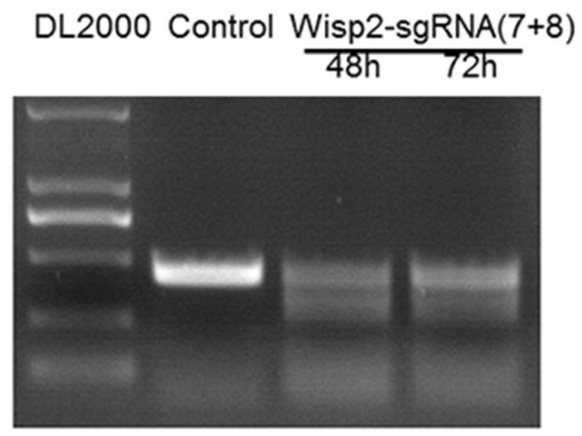

d

Wisp2 sgRNA8

Wisp2 sgRNA7

GGTTTCACCTGCCTGCCGCTGTGCAGTGAGGATGTGCGGCTGCCCAGCTGGGACTGCCCACGCCCCAGGAGAATACAGGTGCCAGGAAGGTGCTGCCCCWT GGTTTCACCTGCCTGCCGCTGTGGAAGG - - OGCTGCCCC\#1 GGTTTCACCTGCCTGCCGCTGTAGGTTTCACCTGCCTGCC-1..-CAGCTGGGACTGCCCACGCCCCAGGAGAATACAGGTGCCAGGAAGGTGCTGCCCC \#6 GGTTTCACCTGCC - ACGCCCCAGGAGAATACAGGTGCCAGGAAGGTGCTGCCCC \#7 GGTTTCACCTGCCTGCCGCTGTGGCGAAGAGGATGTGCGGCTGTGCAACTGGCACTGCCCCCAACTGGCACTGCCCCOGCACAATAAGAATGCCGGAGCC \#8 GGTTTCACCTGCCTGCCGCTGTTGCAGTGAGGATGTGCGGCTGCCCAGCTGGGACTGCCCACGCCCCAGGAGAATACAGGTGC- GGTTTCACCTGCCTGCCGCTGTGCGAA-GAGGATGTGCGGCTGTGCAACTGGCACTGCCCCCGCACAATAAGAATGCCGGAGCCAGGAGCGGGCTGCACC\#13 GGTTTCACCTGCCTGCCGCTGTG-- GAGGTGCTGCCC\#20

e

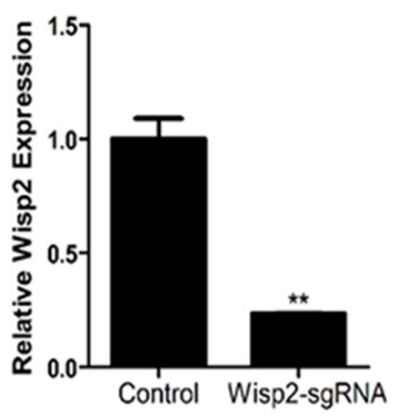

f

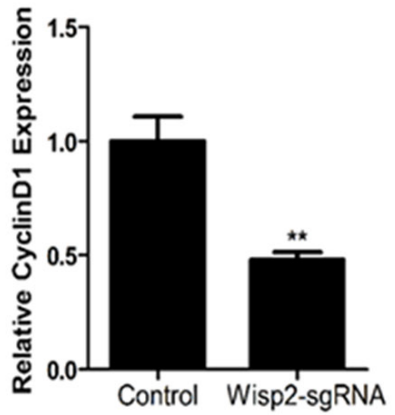

g

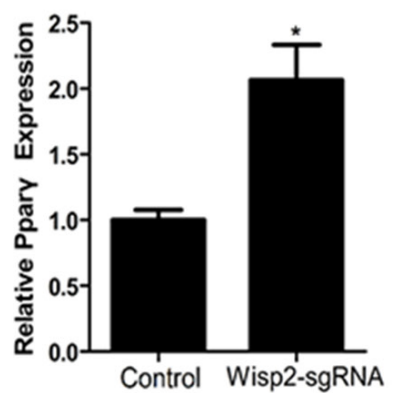

Figure 2: Establishment of Wisp2 knockdown cells by Crispr/Cas9 system. (a) Sketch of the "all in one" lentivirus expression vector containing Cas9 and sgRNAs (Lenti.sgRNAs.Cas9). SgRNAs insertion sites are highlighted in blue. (b and c) High cutting effiency of lentivirus against Wisp2 in C3H10T1/2 cells. PCR products were amplified and subjected to T7EN1. Control, C3H10T1/2 infected with basic vector; $\operatorname{sgW}(7+8)$, cells infected with lentivirus against Wisp2 containing $\operatorname{sgW}(7+8)$; supernatants containing lentivirus were collected 48 hours and 72 hours after the transfection. (d) Sequences of modified Wisp2 detected in C3H10T1/2 cells. 8 of 15 TA clones from the PCR products were analyzed by DNA sequencing and displayed in the images. The PAM sequences are highlighted in green; the targeting sequences are highlighted in red; the mutations are highlighted in blue; deletion (-); insertions $\left({ }^{\wedge}\right)$, lower case; ${ }^{*}$ : clone number. (e-g) QRT-PCR analysis of the expression of Wisp2, CyclinD1 and Ppary in C3H10T1/2 cells infected with lentivirus against Wisp2. CyclinD1 and Ppary were used as positive controls. Gene expression level of the control samples normalized to 36B4 was set as 1. Results are shown as mean \pm S.E.M. of 3 independent experiments. “**”, $\mathrm{p}<0.01$, “*”, $\mathrm{P}<0.05$. 
a

\section{DL2000 NC BMSCs Control Wisp2-sgRNA}

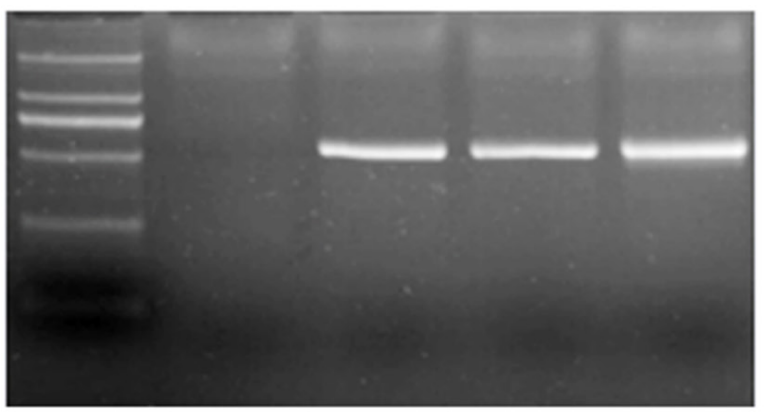

C

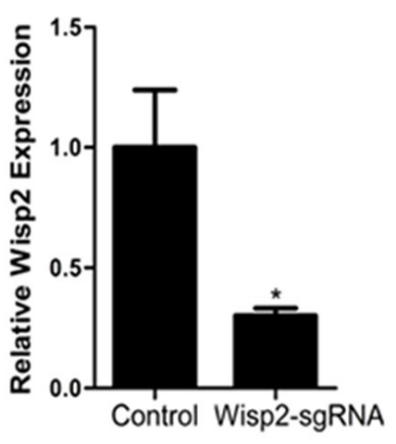

$\mathbf{f}$
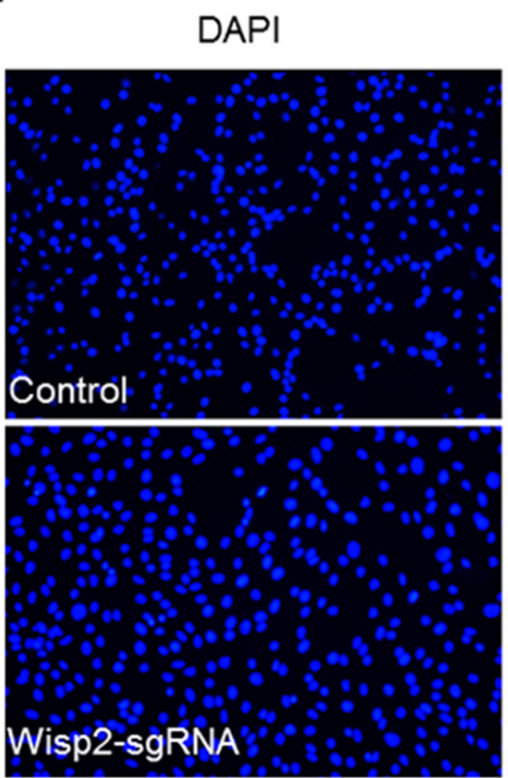

b

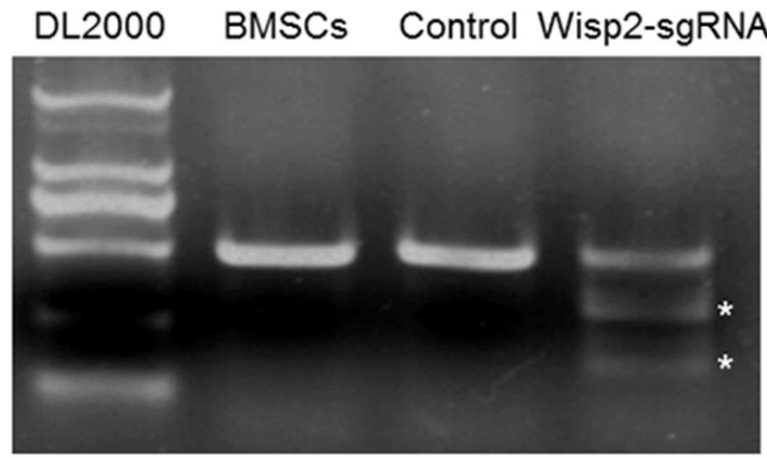

e

Control Wisp2-sgRNA

Cxcr4

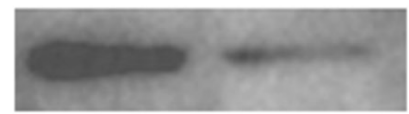

Wisp2

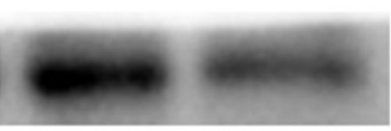

Gapdh

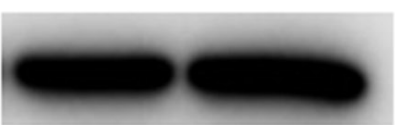

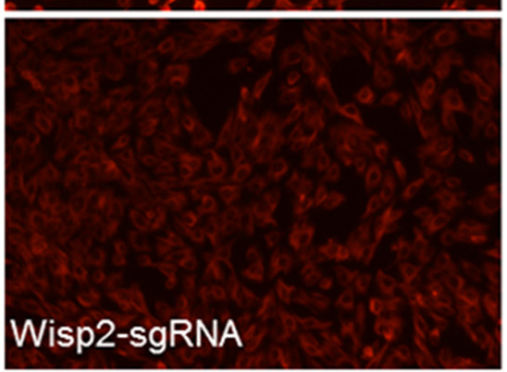

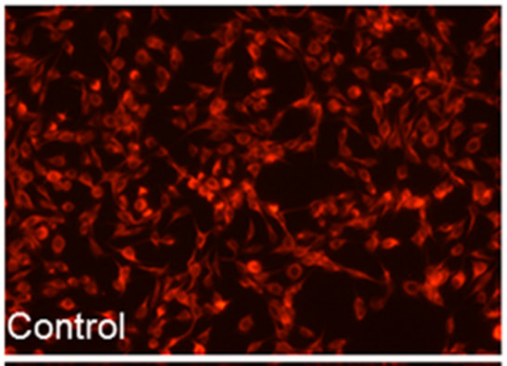

Figure 3: Down-regulation of Cxcr4 in Wisp2 genetically modified cells. (a) PCR products of the targeted Wisp2 from BMSCs infected with lentivirus against Wisp2 (Wisp2-sgRNA) or lentivirus without sgRNAs (Control). (b) Cas9 mediated on-target cleavage of Wisp2 by T7EN1. PCR products were amplified and subjected to T7EN1. Samples with cleavage bands were marked with an asterisk ${ }^{\text {c**”. }}$. (c and d) QRT-PCR analysis of the expression of Wisp2 and Cxcr4 in BMSCs infected with lentivirus against Wisp2. The error bars indicated the \pm S.E.M. of at least three independent experiments, “*”, $p<0.05$. Gene expression level of the control samples normalized to Gapdh was set as 1. (e) Wisp2 and Cxcr4 protein expression levels were depressed in the Wisp2-sgRNA-BMSCs. Total cellular protein was analyzed by immunoblotting for Gapdh. $20 \mu \mathrm{g}$ of total protein per lane. (f) Immunofluorescence showed that Cxcr4 was reduced in the Wisp2-sgRNA-BMSCs. Blue, DAPI; Red, Cxcr4. 
tail vein into rats that underwent liver injury by a 2-AAF/ $\mathrm{PH}$ treatment. GFP was used as a reporter to detect the transplanted BMSCs. Two days later, BMSCs were found around the blood vessels, and their abundance increased at day 10 . This finding is consistent with previous reports $[10,28]$.

The most novel observation in this report is the delayed liver repair in the Wisp2-sgRNA rats compared to the control rats after the liver injury. These results identified an unexpected role of Wisp2 directed BMSCs in regulating liver repair. Wisp2 has been reported to be involved in cell proliferation, migration, wound healing and angiogenesis [29]. Subsequently, a study has demonstrated that the inhibition of Wisp2 at the mRNA and protein levels can effectively suppress the proliferation of cells [30]. These findings are consistent with our results, as we found that more PCNA staining signal in control group, in contrast, IL- 6 and $\alpha$-SMA were increased in Wisp2-sgRNA group. We further showed that the expression of Cxcr4 was largely decreased in the Wisp2-sgRNA rats and the Wisp2-sgRNA cells.

a

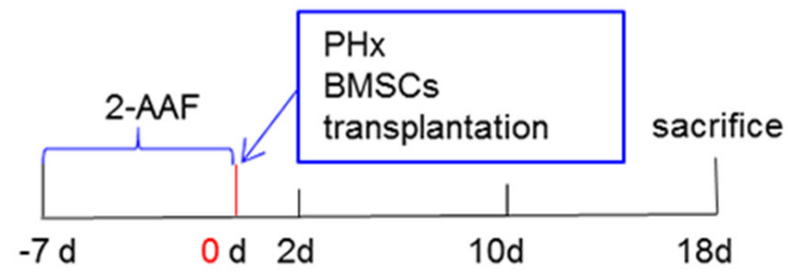

b

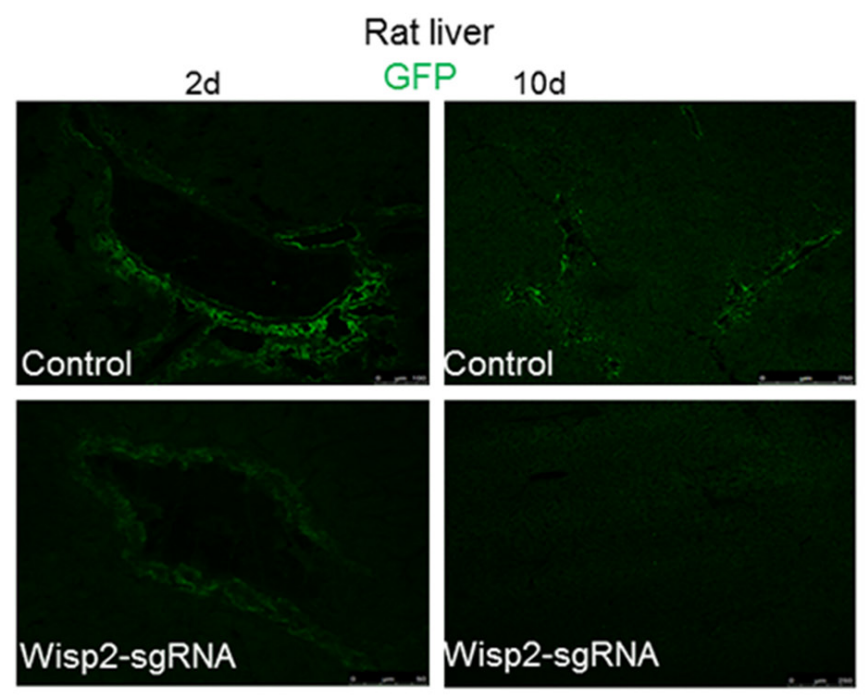

Intriguingly, BMSCs express relatively high levels of Wisp2 and Cxcr4 [31].

Cxcr4, which is a G-protein-coupled receptor, is highly important due to its critical role in development regulation, hematopoiesis, angiogenesis, stem cell migration and wound healing [32]. Ding has demonstrated that after an acute liver injury, Cxcr7 up-regulation in liver sinusoidal endothelial cells acts in conjunction with Cxcr4 to induce transcription factor Id1, which deploys pro-regenerative angiogenic factors and triggers regeneration. This phenomenon was abrogated by genetic silencing of either Cxcr4 or Cxcr7 [28]. Dmitriev has proposed that Dux 4 controlled the cellular migration of MSCs through the Cxcr4 receptor. Adding Cxcr4 antibody to the culture medium completely abolished the effect of Dux 4 overexpression on cell migration [32]. In addition, it has been reported that the overexpression of Cxcr4 improved the efficiency of infused MSCs homing toward the damaged tissues [33]. Besides, many studies have demonstrated that $\mathrm{Cxcr} 4$ played a pivotal role in mobilizing BMSCs to the damaged tissues and promoting wound

\section{C}

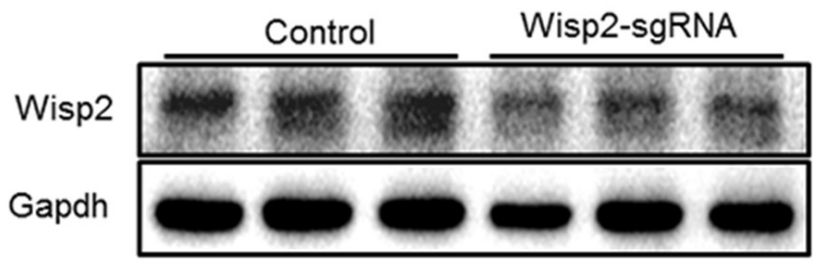

d

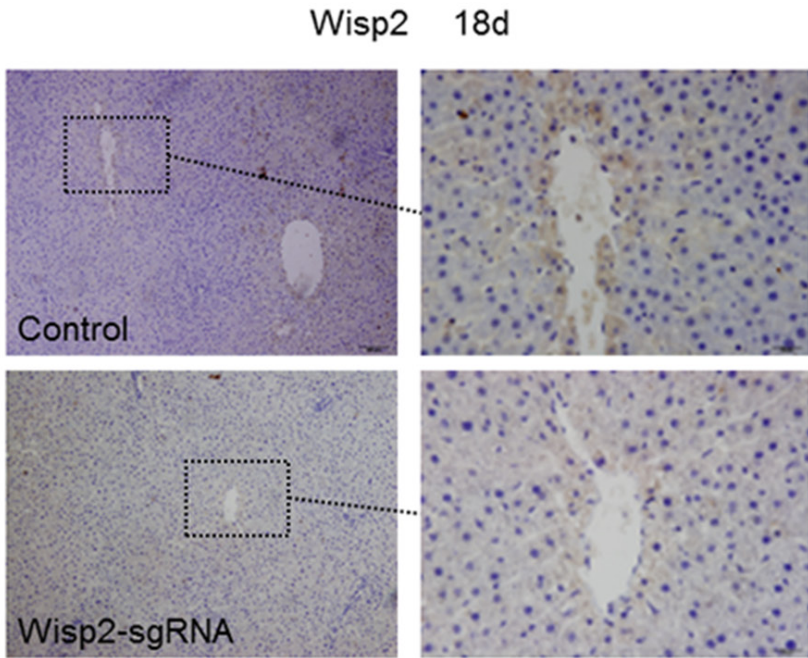

Figure 4: Wisp2 was disrupted in rat livers after 2-AAF/PH. (a) Seven days after the intragastric administration of 2-AAF, the rats underwent $\mathrm{PH}$ and were transplanted with $1.8 \times 10^{6}$ Wisp2-sgRNA-BMSCs or equal amounts of control BMSCs and then sacrificed and livers collected as indicated in the scheme. (b) Representative images showing homing BMSCs to the injured liver. (c and d) Wisp2 expressed in rat livers. Expression of Wisp2 was detected in rat livers 18 days post-transplantation by Immunoblot analysis (c). $40 \mu \mathrm{g}$ of total protein per lane. (d) Immunostaining was performed. Original magnification: 10× (Left panel) and 40× (right panel). 
a

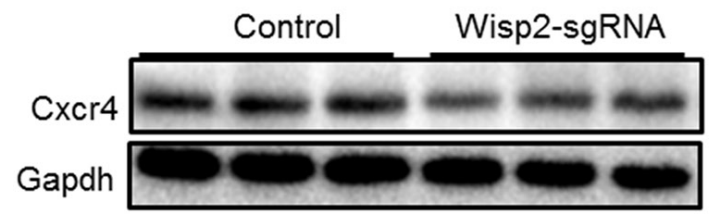

b

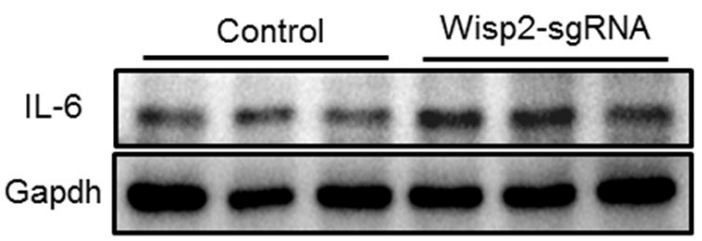

d

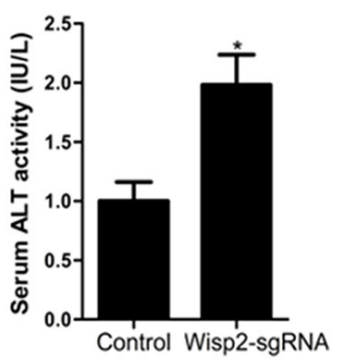

f
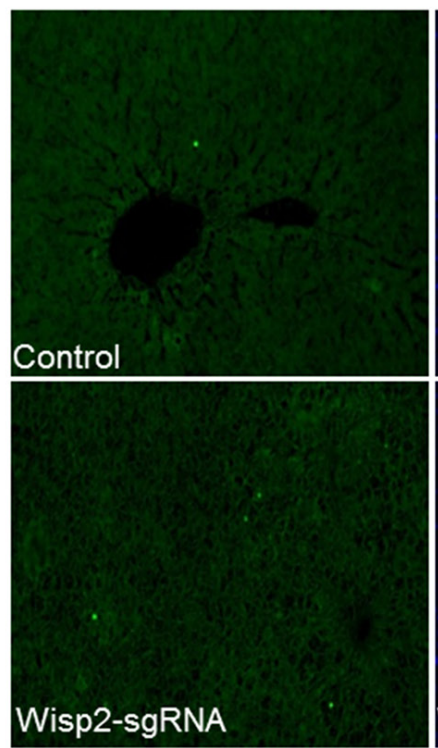

C

HE

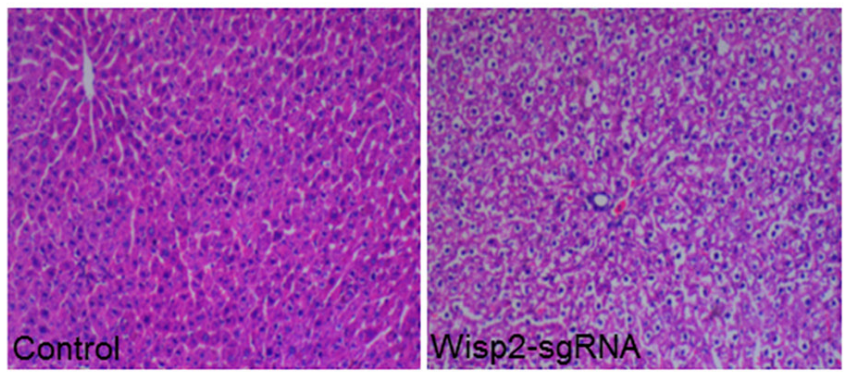

PCNA
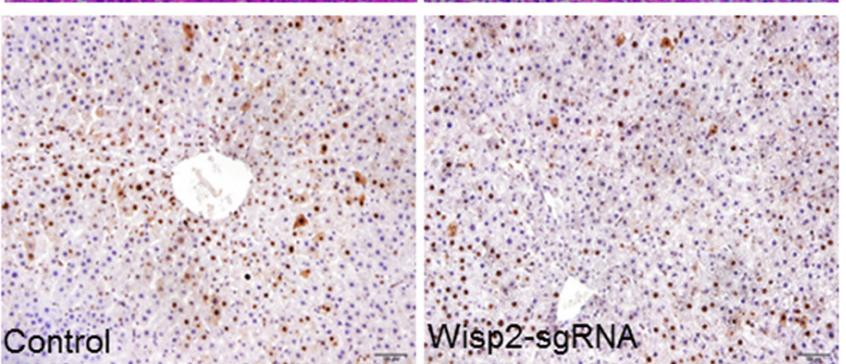

a-SMA

\section{Control}
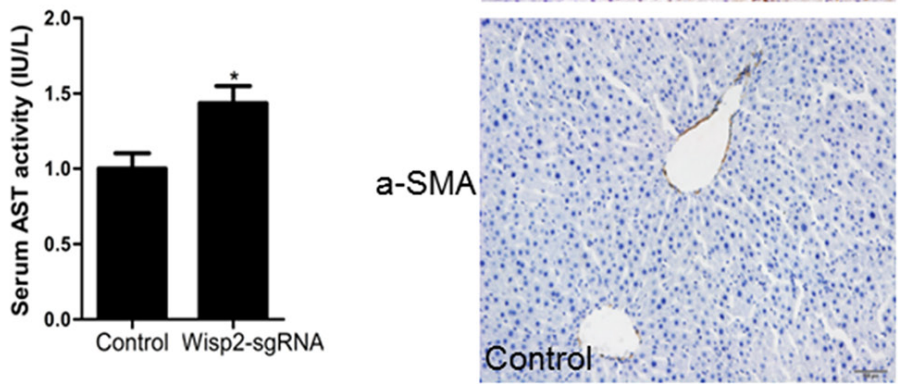

Figure 5: Cxcr4 was decreased and repair capability was reduced in lentiviral Wisp2 disrupted BMSC transplantation

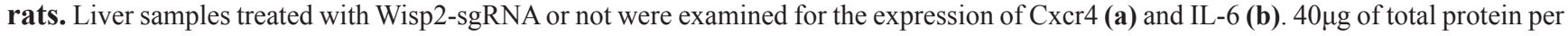
lane, as indicated by western analysis 18 days after transplantation. (c) Paraffin- embedded sections from livers obtained 18 days after the transplantation were stained with H\&E and PCNA or $\alpha$-SMA. The Wisp2-sgRNA group showed more extensive liver damage and cellular swelling (20×). Serum ALT (d) and AST (e) levels were assessed to determine the degree of liver injury. The control group was set as 1. Asterisks indicate significant differences, “"”, $\mathrm{P}<0.05$. (f) Representative micrographs of TUNEL staining of liver tissue. Liver sections collected 18 days after BMSCs transplantation were stained with FITC-conjugated TUNEL to identify apoptotic cells. The results showed that disrupted Wisp2 incresed the levels of apoptosis caused by 2-AAF/PH. Original magnification: $20 \times$. 


\section{a}
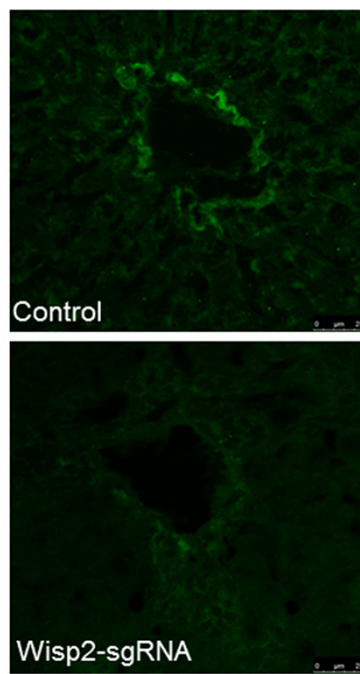

b
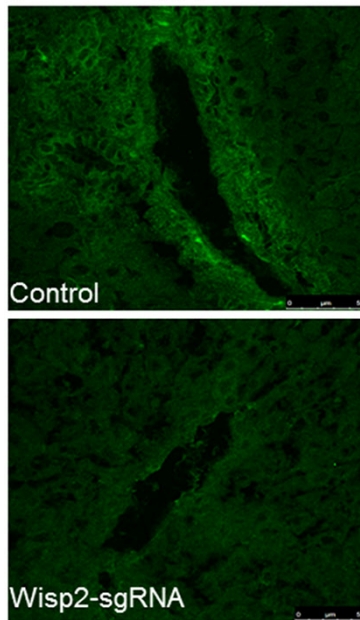

Control
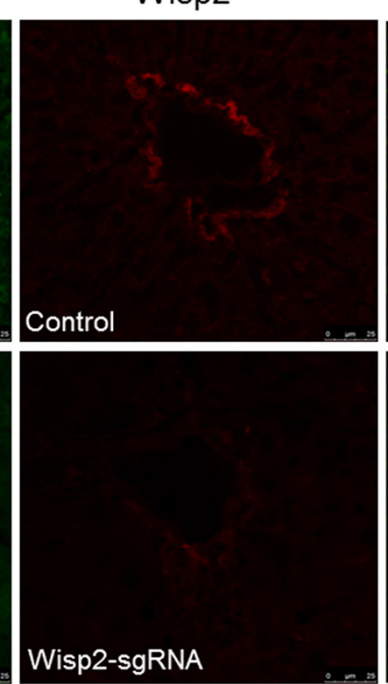

Cxcr4

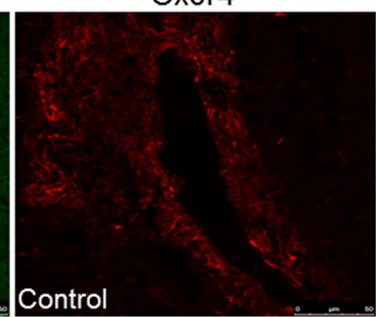

Wisp2-sgRNA
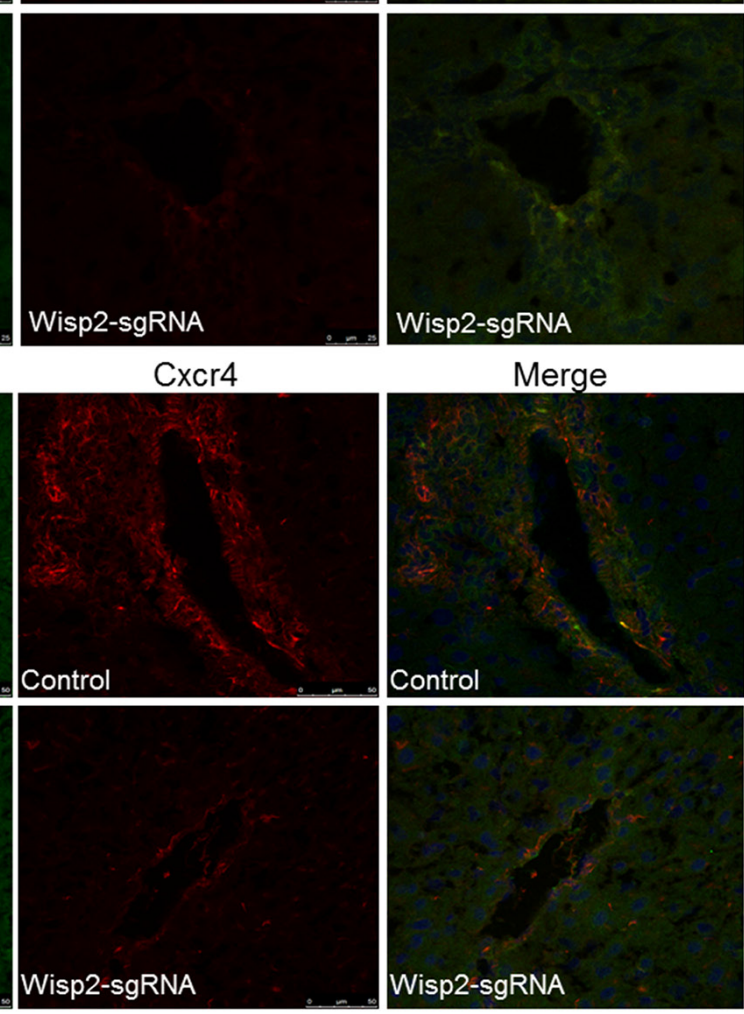

C

GFP
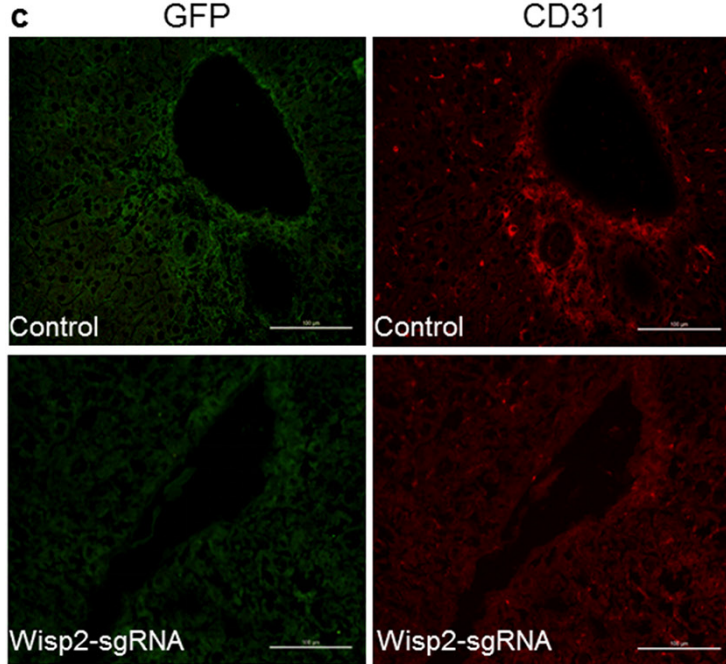

Control
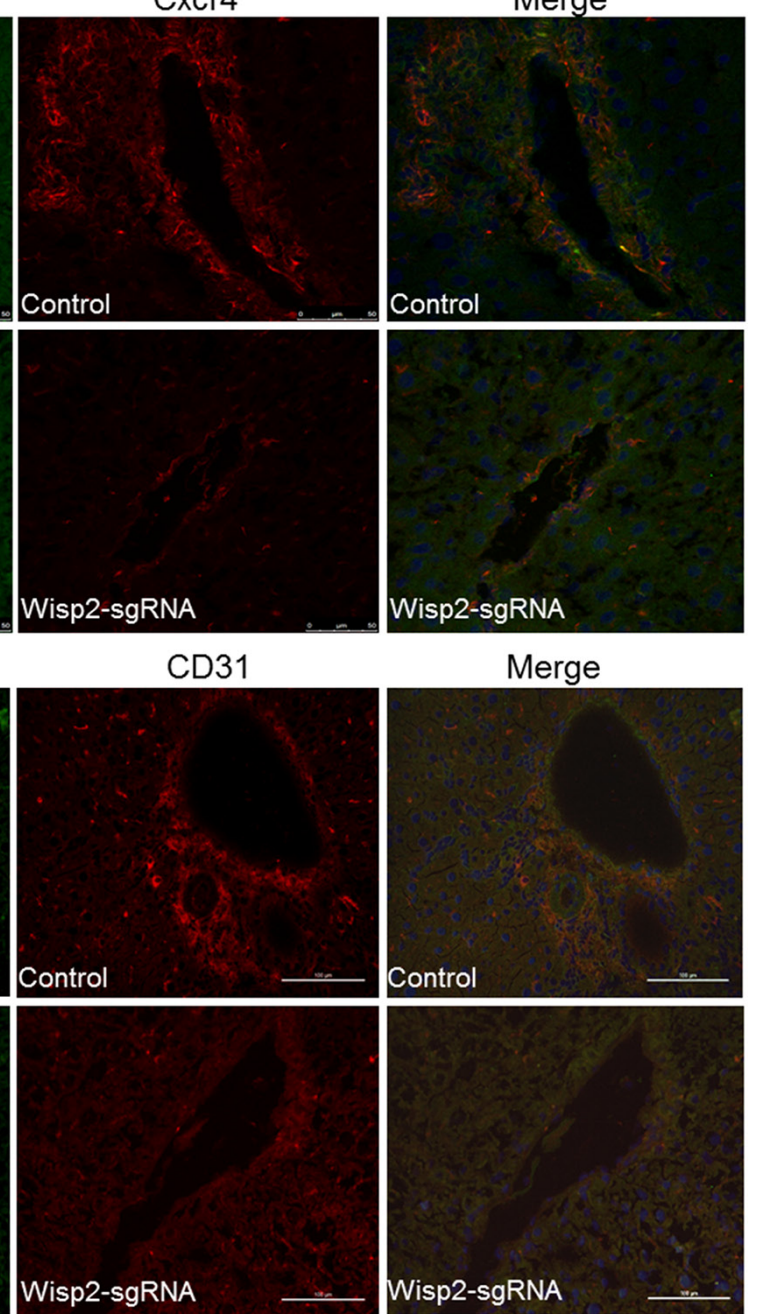

Merge

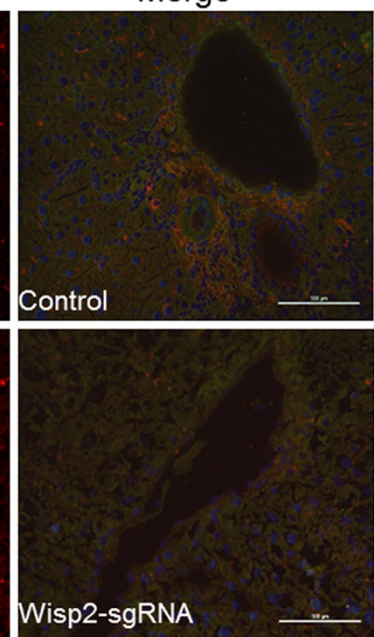

Figure 6: Wisp2 was required for BMSC migration and differentiation to endothelial cells. Significantly decreased expression of Wisp2 and Cxcr4 in rat livers after the Wisp2-sgRNA-BMSC transplanted 18 days, the expression of Wisp2 (a) and Cxcr4 (b) were determined in liver sections. Liver cyrosections were stained for GFP (Green fluorescence) and Wisp2/Cxcr4 (Red fluorescence). Images were merged with DAPI (Blue fluorescence) staining reveal the nuclei. (c) Expression of the endothelial cell marker CD31 (Red fluorescence) was also examined in the liver sections by immunostaining. The expression of CD31 was reduced in the Wisp2-sgRNA BMSCs transplanted rat livers. 
Table 2: Primers for PCR and qRT-PCR

\begin{tabular}{|c|c|c|}
\hline Genes & primers $\left(5^{\prime}-3^{\prime}\right)$ & Accession number \\
\hline r-Wisp2 & $\begin{array}{l}\text { TGCCTGCTCGGGAAGTA } \\
\text { CGCTTGATTTGGGTGTTTA }\end{array}$ & NM_031590.1 \\
\hline $\mathrm{r}-\mathrm{Cxcr} 4$ & $\begin{array}{l}\text { GCATCGTCATCCTGTCCTGT } \\
\text { ACGCTCTCGAACTCACATCC }\end{array}$ & NM_022205.3 \\
\hline r-Wisp2-test & $\begin{array}{l}\text { TCCTGGTCCTCTTTCTCC } \\
\text { AAGGTAGGGCTGGTTATG }\end{array}$ & NC_005102.4 \\
\hline $\mathrm{r}-\mathrm{Cxcr} 7$ & $\begin{array}{l}\text { CAGCACCTCCAGCTATAAGAA } \\
\text { TGGCGAGCAGGAAGTAGA }\end{array}$ & NM_053352.1 \\
\hline $\mathrm{m}-\mathrm{Cxcr} 4$ & $\begin{array}{l}\text { AGCATGACGGACAAGTACC } \\
\text { GATGATATGGACAGCCTTACAC }\end{array}$ & NM_009911.3 \\
\hline $\mathrm{m}-$ Wisp 2 & $\begin{array}{l}\text { TGACAGACGCTCCTGATCTCCA } \\
\text { CAGCAAGAAAGACCTCCATCCC }\end{array}$ & NM_016873.2 \\
\hline m-Wisp2-test & $\begin{array}{l}\text { GGAGAAACCGAGGCAAGATGTGA } \\
\text { GCACTCACTCACCTTGGGCTGA }\end{array}$ & NC_000068.7 \\
\hline $\mathrm{m}-C y c l i n D 1$ & $\begin{array}{l}\text { TCAAGTGTGACCCGGACTG } \\
\text { ATGTCCACATCTCGCACGTC }\end{array}$ & NM_007631.2 \\
\hline $\mathrm{m}-$-Ppary & $\begin{array}{l}\text { TGGGTGAAACTCTGGGAGATTC } \\
\text { AGAGGTCCACAGAGCTGATTCC }\end{array}$ & NM_001127330.2 \\
\hline $\mathrm{m}-\mathrm{Cxcr} 7$ & $\begin{array}{l}\text { GCGTATCAAAGCCAGCACC } \\
\text { CAACAGCCTTACACCTCCC }\end{array}$ & NM_001271607.1 \\
\hline $\mathrm{m}-36 B 4$ & $\begin{array}{l}\text { TGGAGACAAGGTGGGAGCC } \\
\text { CACAGACAATGCCAGGACGC }\end{array}$ & NM_007475.5 \\
\hline
\end{tabular}

healing [28, 32, 34-36]. Consequently, the regulation of Cxcr4 expression was expected to influence the directional homing of infused BMSCs. Thus, we proposed that Wisp2 malfunction causing Cxcr4 downregulation might be the major mechanism for delayed liver repair. However, we would like to mention that another study implicated that the constitutive activation of Cxcr4 in LSECs by chronic injury established a pro-fibrotic vascular niche, activating adjacent myofibroblast cells and provoking fibrogenesis [28].Therefore, a more comprehensive investigation of Cxcr4 is needed in the future.

Additionally, the differentiation of BMSCs in vivo is also a matter of concern. Studies investigating BMSCs have shown their capability to differentiate into many specific cell types [37-39]. CD31, also named PECAM-1, was a specific marker of endothelial cells [40]. Several studies have suggested that CD31 may be involved in the process of angiogenesis. Studies of human microvascular endothelial cells grown in a three-dimensional culture in the presence of factors that promote angiogenesis support this concept. In these studies, the exposure to antiCD31 antibodies or soluble CD31 protein was shown to inhibit the formation of normal multicellular tubes [41]. In this study, we noticed that while Wisp2 was knocked down, the reduction of Cxcr4 inhibited the homing of BMSCs towards to the injured liver and simultaneously decreased the number of $\mathrm{CD}^{+} 1^{+}$endothelial cells by immunofluorescence. The result is consistent with previous reports which showed that BMSCs could be recruited at the sites of neovascularization through the paracrine regulation of their angiogenic properties [42]. Furthermore, we found that BMSCs could differentiate into $\mathrm{CK} 19^{+}$cell, but hardly into $\mathrm{vWF}^{+}$cells. Thus, we suggest that knocking down Wisp2 repressed endothelial cell proliferation and angiogenesis and then delayed liver repair.

\section{MATERIALS AND METHODS}

\section{Ethics statement}

Investigation has been conducted in accordance with the ethical standards and according to national and international guidelines and has been approved by Huazhong Agriculture University for the care and use of laboratory animals.

\section{Cell culture}

NIH3T3, Hep1-6 and 293T cells were cultured with $10 \%$ fetal bovine serum (FBS, Thermo Fisher, Waltham, USA) in H-DMEM (Thermo Fisher). C3H10T1/2 cells were cultured with $10 \%$ FBS in EBSS-MEM (Thermo 
Fisher) including 1\% NEAA (Solarbio, beijing, USA). Rat BMSCs (RASMX-01201) were purchased from Cyagen Biosciences and cultured in DMEM supplemented with $10 \%$ fetal bovine serum, $100 \mathrm{IU} / \mathrm{mL}$ penicillinstreptomycin, and $2 \mathrm{mM}$ glutamine. Cells were all incubated at $37^{\circ} \mathrm{C}$ in a humidified incubator containing $5 \% \mathrm{CO}_{2}$.

\section{Animal model}

SD rats, 5-6 weeks of age (170-180 g), were purchased from Hubei Provincial Center for Disease Control and Prevention. These animals were bred in-house and maintained on standard laboratory chow and a daily 12-hour light/12-hour dark cycle. Each rat was given 20 $\mathrm{mg} / \mathrm{kg}$ 2-Acetylaminofluorene(2-AAF, Sigma Chemicals, CA, USA) dissolved in PEG400 (Servicebio, Wuhan, China) via an intragastric administration once a day for 1 week, and then, two-thirds of their livers were removed [43]. Then, the rats were immediately divided into two groups and underwent transplantation with $1.8 \times 10^{6}$ Wisp2sgRNA BMSCs or control BMSCs in a total volume of $500 \mu \mathrm{l}$ by tail vein injection.

\section{Cas9/ sgRNA efficiency test in cells}

The sgRNAs were designed online as shown in Table 1 and cloned into Px330 or a lentiviral plasmid. The transfection procedure was carried out using lipofectamine 2000 reagent (Invitrogen, Carlsbad, USA) according to the manufacturer's instructions. Briefly, the cells were transfected with Px330 containing the sgWisp2 plasmid or basic plasmid by lipofectamine 2000 in a 6-well culture plate. Then, 48 hours after the transfection, genomic DNA was extracted from the cells with a lysis buffer. Next, the DNA was precipitated with alcohol and sodium acetate. Subsequently, a T7EN1 (NEB, New England Biolabs, USA) cleavage assay was performed as described by Shen et al [44]. Briefly, the targeted fragments from the genomic DNA were amplified by a $2 \times$ Taq PCR Mix (Ald lab, Beijing, China) and purified using a TYIAN Quick Midi Purification Kit (Tiangen, Beijing, China). The primers used to amplify the Wisp2 targeted fragments are listed in Table 2. The purified PCR products were denatured and re-annealed in NE Buffer 2 (NEB) using a thermocycler (Long Gene, A200, China). The PCR products were digested with T7EN1 for $30 \mathrm{~min}$ at $37^{\circ} \mathrm{C}$ and then separated on a $1.5 \%$ agarose gel. The PCR products with mutations detected by the T7EN1 cleavage assay were then sub-cloned into a PMD-18T vector (Takara, Japan). The colonies were randomly selected and sequenced with specific primers listed in Table 2.

\section{Lentivirus generation and cell infection}

The resulting shuttle vectors with or without $\operatorname{sgW}(7+8)$ were mixed with a packaging mix (pMD2.G and PsPAX2). Then, the mixed vectors were added to the opti-MEM (Invitrogen, Carlsbad, USA) culture medium and transfected into the 293 T packaging cell line. Recombinant lentivirus expressing both GFP and sgRNAs of Wisp2 were harvested and filtered through a $0.45 \mu \mathrm{m}$ filter (Millipore, USA) after 48 hours or 72 hours. For the infection, the cells were transduced with lentiviruses in 5\% FBS growth media supplemented with polybrene $(8 \mu \mathrm{g} / \mathrm{ml})$. The cells were incubated overnight with the lentivirus and cultured in fresh 5\% FBS growth media for an additional 3 days. Subsequently, the cells were cultured in 10\% FBS growth media. Finally, we detected the cutting efficiency as described above.

\section{Real-time quantitative PCR}

Total RNA was isolated and purified using RNAiso plus (Takara, Japan) and transcribed into cDNA using the first strand cDNA synthesis kit (TOYOBO, Japan). The cDNA was diluted for the real-time quantitative PCR (qRT-PCR), and the samples were run in a $10 \mu$ reaction system using the SYBR GREEN qPCR mix (TOYOBO, Japan). The data were detected using the ABI CFX Connect TM Real-Time PCR Detection System (ABI, USA). The level of mRNA in each sample was normalized with respect to $36 \mathrm{~B} 4$ as the internal standard.

\section{Western blotting}

The cells were washed with cold PBS, lysed (Beyotime, Shanghai, China) on ice for a few minutes, and centrifuged at $12,000 \mathrm{~g}$ for $5 \mathrm{~min}$ at $4^{\circ} \mathrm{C}$, and the supernatants containing the total proteins were collected. The supernatants were subjected to SDS-PAGE and immunoblotting. In total, proteins were separated on a $15 \%$ polyacrylamide gel, followed by transferring onto a PVDF membrane (Millipore, Billerica, MA, USA). The membrane was blocked for 2 hours with 5\% skim milk (Bio sharp, China) and incubated overnight with the anti-Wisp2 (Bioss, bs-5100s), anti-Cxcr4 (Santa Cruz, sc-53534) and anti-Gapdh (Santa Cruz, sc-293335) antibodies. After 3 washes, the secondary antibody was added at a 1:5,000 dilution and incubated at room temperature for 1.5 hours. After 4 washes, the membrane was exposed using western bright TM ECL (Juneng, K-12045-D10) in the imaging system (SYNGENE, G: Box). The protein amount was normalized to the amount of Gapdh as the internal control.

\section{Serum transaminase levels and histological analysis}

The serum aspartate aminotransferase (AST) and alanine aminotransferase (ALT) levels in the rat blood were measured with assays purchased from Nanjing Jiancheng (C010-2, C009-2). For the histologic assessment, the livers were fixed in $4 \%$ formaldehyde for 
24 hours and embedded in paraffin. Liver sections $(4 \mu \mathrm{m})$ were deparaffinized and fixed. The sections were stained with $\mathrm{H} \& \mathrm{E}$.

\section{Immunostaining and histological analysis of liver cryosections}

To collect tissues for the histological analysis, the rat livers were snap-frozen in OCT. For the immunofluorescence microscopy, the liver sections $(6 \mu \mathrm{m})$ were blocked ( $10 \%$ goat serum $/ 1 \%$ Triton $\mathrm{X}-100)$ and incubated in the following primary antibodies: anti-Wisp2 $(10 \mu \mathrm{g} / \mathrm{ml}$, Santa Cruz, sc-514070), anti-Cxcr4, anti-GFP

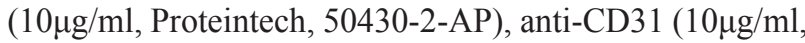
Santa Cruz, sc-376764), anti-PCNA $(10 \mu \mathrm{g} / \mathrm{ml}$, Santa Cruz, sc-25280), anti- $\alpha$-SMA (10 $\mu \mathrm{g} / \mathrm{ml}$, Servicebio, GB13044), anti-CK19 $(10 \mu \mathrm{g} / \mathrm{ml}$, Santa Cruz, sc-376126) and antivWF $(10 \mu \mathrm{g} / \mathrm{ml}$, Santa Cruz, sc-53466). After incubation in fluorophore-conjugated secondary antibodies $(2.5 \mu \mathrm{g} /$ $\mathrm{ml}$, Invitrogen, A-11034, A-21424), the sections were counterstained with DAPI (Invitrogen). Finally, confocal microscope and fluorescent microscope were used to observe the results.

\section{TUNEL staining}

In Situ Cell Death Detection Kit, Fluorescein (cat no.11684795510) was purchased from Roche and terminal deoxynucleotidyl transferase 2-deoxyuridine, 5-triphosphate nick end labeling (TUNEL) staining was performed with the manufacturer's instructions.

\section{Statistical analysis}

All data are presented as the mean \pm S.E.M. of at least three separate experiments. Differences between groups were tested for statistical significance using Student's two-tailed $t$-tests. Statistical significance was set at $\mathrm{P}<0.05\left(^{*}\right), \mathrm{P}<0.01\left(^{* *}\right)$.

\section{CONCLUSIONS}

Although much remains unknown, our results confirm a critical role of Wisp2 in promoting liver repair. Moreover, our studies demonstrate that knockdown of Wisp2 repressed Cxcr4 expression and decelerated liver repair. Therefore, both Wisp2 and Cxcr4 participate in the promotion of liver repair.

\section{Abbreviations}

2-AAF: 2-Acetylaminofluorene; $\alpha$-SMA: $\alpha$-smooth muscle actin; All in one: One plasmid which contains both GFP, Cas9 and sgRNAs; ALI: Acute liver injury; ALF: Acute liver failure; ALT: Alanine transaminase; AST: Aspartate amino transferase; BMSC: Bone marrow-derived mesenchyme stem cell; CK19: Cytokeratin 19; CRC:
Colorectal cancer; CRISPR/Cas9: Clustered regularly interspaced short palindromic repeats (CRISPR)-associated protein -9 nuclease; Cxcr4: C-X-C chemokine receptor type 4; GFP: Green fluorescence protein; IL-6: Interleukin-6; MSCs: Mesenchyme stem cells; PCNA: Proliferating cell nuclear antigen; TUNEL: Terminal deoxynucleotidyl transferase 2-deoxyuridine, 5-triphosphate nick end labeling; vWF: von Willebrand factor; Wisp2: Wnt-1 inducible signaling pathway protein 2 .

\section{Author contributions}

Conception and design: LZ, XL, HL; Acquisition of data: DQ, YY; SgRNA design for the CRISPR/Cas9 system and vectors construction: $\mathrm{BH}, \mathrm{XH}$; Analysis and interpretation of data: WZ, SL, XH; Writing, review, and/ or revision of the manuscript: LZ, DQ, DD.

\section{ACKNOWLEDGMENTS}

We gratefully acknowledge the excellent technical assistance of Shengsong Xie and Tongxing Song. We thank the people at the physiological laboratory and pathology laboratory for scientific discussions.

\section{Availability of data and material}

The datasets used and/or analyzed during the current study are available from the corresponding author on reasonable request.

\section{CONFLICTS OF INTEREST}

The authors declare that they have no competing interests.

\section{FUNDING}

This work was supported by National Key R\&D Plan No. 2017YFA0103200 \& No. 2017YFA0103202, the Fundamental Research Funds for the Central Universities (2662017PY106 and 2662016PY087), 1000 plan project and HZAU Startup funds to LZ.

\section{REFERENCES}

1. Jemal A, Bray F, Center MM, Ferlay J, Ward E, Forman D. Global cancer statistics. CA Cancer Journal for Clinicians. 2011; 61:69-90.

2. Torre LA, Bray F, Siegel RL, Ferlay J, Lortet-Tieulent J, Jemal A. Global cancer statistics, 2012. CA Cancer Journal for Clinicians. 2015; 65:87-108.

3. McDowell TD, Stevens RD, Gurakar A. Acute liver failure: a management challenge for the practicing gastroenterologist. Gastroenterology \& Hepatology. 2010; 6:444-450. 
4. Oertel M, Shafritz DA. Stem cells, cell transplantation and liver repopulation. Biochimica Et Biophysica Acta. 2008; 1782:61-74.

5. Oh SH, Witek RP, Bae SH, Zheng D, Jung Y, Piscaglia $\mathrm{AC}$, Petersen BE. Bone marrow-derived hepatic oval cells differentiate into hepatocytes in 2-acetylaminofluorene/ partial hepatectomy induced liver regeneration. Gastroenterology. 2007; 132:1077-1087.

6. Mareddy S, Xiao Y, Crawford R. Characterization of bone marrow stem cell (BMSC) clones and their potential application for tissue engineering. Cytometry Part A. 2006:420.

7. Pittenger MF, Mackay AM, Beck SC, Jaiswal RK, Douglas R, Mosca JD, Moorman MA, Simonetti DW, Craig S, Marshak DR. Multilineage potential of adult human mesenchymal stem cells. Science. 1999; 284:143-147.

8. Hang HL, Xia Q. Role of BMSCs in liver regeneration and metastasis after hepatectomy. World Journal of Gastroenterology. 2014; 20:126-132.

9. Hocking AM. The role of chemokines in mesenchymal stem cell homing to wounds. Advances in Wound Care. 2015; 4:623-630.

10. Song YM, Lian CH, Wu CS, Ji AF, Xiang JJ, Wang XY. Effects of bone marrow-derived mesenchymal stem cells transplanted via the portal vein or tail vein on liver injury in rats with liver cirrhosis. Experimental \& Therapeutic Medicine. 2015; 9:1292-1298.

11. Yao Y, Zhang F, Wang L, Zhang G, Wang Z, Chen J, Gao X. Lipopolysaccharide preconditioning enhances the efficacy of mesenchymal stem cells transplantation in a rat model of acute myocardial infarction. Journal of Biomedical Science. $2009 ; 16: 74-84$

12. Cai A, Zheng D, Dong Y, Qiu R, Huang Y, Song Y, Jiang Z, Rao S, Liao X, Kuang J, Dai G, Mai W. Efficacy of atorvastatin combined with adipose-derived mesenchymal stem cell transplantation on cardiac function in rats with acute myocardial infarction. Acta Biochimica Et Biophysica Sinica. 2011; 43:857-866.

13. Ma HC, Shi XL, Ren HZ, Yuan XW, Ding YT. Targeted migration of mesenchymal stem cells modified with Cxcr4 to acute failing liver improves liver regeneration. World Journal of Gastroenterology Wjg. 2014; 20:14884-14894.

14. Lee H, Park JB, Lee S, Baek S, Kim H, Kim SJ. Intraosseous injection of donor mesenchymal stem cell (MSC) into the bone marrow in living donor kidney transplantation; a pilot study. Journal of Translational Medicine. 2013; 11:96-103.

15. Cervello M, Giannitrapani L, Labbozzetta M, Notarbartolo M, D’Alessandro N, Lampiasi N, Azzolina A, Montalto G. Expression of Wisps and of their novel alternative variants in human hepatocellular carcinoma cells. Annals of the New York Academy of Sciences. 2004; 1028:432-439.

16. Pennica D, Swanson TA, Welsh JW, Roy MA, Lawrence DA, Lee J, Brush J, Taneyhill LA, Deuel B, Lew M. Wisp genes are members of the connective tissue growth factor family that are up-regulated in Wnt-1-transformed cells and aberrantly expressed in human colon tumors. Proceedings of the National Academy of Sciences. 1998; 95:14717-14722.

17. Grünberg JR, Hammarstedt A, Hedjazifar S, Smith U. The novel secreted adipokine WNT1-inducible signaling pathway protein 2 (WISP2) is a mesenchymal cell activator of canonical WNT. Journal of Biological Chemistry. 2014; 289:6899-6907.

18. Schutze N, Noth U, Schneidereit J, Hendrich C, Jakob F. Differential expression of CCN-family members in primary human bone marrow-derived mesenchymal stem cells during osteogenic, chondrogenic and adipogenic differentiation. Cell Communication and Signaling. 2005; 3:5-17.

19. Hammarstedt A, Hedjazifar S, Jenndahl L, Gogg S, Grünberg J, Gustafson B, Klimcakova E, Stich V, Langin D, Laakso M, Smith U. Wisp2 regulates preadipocyte commitment and Ppary activation by BMP4. Proceedings of the National Academy of Sciences of the United States of America. 2013; 110:2563-2568.

20. Frewer K, Sanders A, Owen S, Hargest R, Jiang W. P30. Wisp2 and colorectal cancer cell invasiveness. European Journal of Surgical Oncology. 2012; 38:1114.

21. Hsu PD, Lander ES, Zhang F. Development and applications of CRISPR-Cas9 for genome engineering. Cell. 2014; 157:1262-1278.

22. Yagi H, Parekkadan B, Suganuma K, Soto-Gutierrez A, Tompkins RG, Tilles AW, Yarmush ML. Long-term superior performance of a stem cell/hepatocyte device for the treatment of acute liver failure. Tissue Engineering Part A. 2009; 15:3377-3388.

23. Christ B, Dollinger MM. The generation of hepatocytes from mesenchymal stem cells and engraftment into the liver. Current Opinion in Organ Transplantation. 2010; 16:69-75.

24. Wu MY, Xie X, Xu ZK, Xie L, Chen Z, Shou LM, Gong FR, Xie YF, Li W, Tao M. PP2A inhibitors suppress migration and growth of PANC-1 pancreatic cancer cells through inhibition on the $\mathrm{Wnt} / \beta$-catenin pathway by phosphorylation and degradation of $\beta$-catenin. Oncology Reports. 2014; 32:513-522.

25. Banerjee S, Saxena N, Sengupta K, Tawfik O, Mayo MS, Banerjee SK. Wisp2 gene in human breast cancer: estrogen and progesterone inducible expression and regulation of tumor cell proliferation. Neoplasia. 2003; 5:63-73.

26. Wannemuehler TJ, Manukyan MC, Brewster BD, Rouch J, Poynter JA, Wang Y, Meldrum DR. Advances in mesenchymal stem cell research in sepsis. Journal of Surgical Research. 2012; 173:113-126.

27. Kitada M, Dezawa M. Parkinson's disease and mesenchymal stem cells: potential for cell-based therapy. Parkinson's disease. 2012; 2012:873706-873715. 
28. Ding BS, Cao Z, Lis R, Nolan DJ, Guo P, Simons M, Penfold ME, Shido K, Rabbany SY, Rafii S. Divergent angiocrine signals from vascular niche balance liver regeneration and fibrosis. Nature. 2014; 505:97-102.

29. Ohkawa Y, Ohmi Y, Tajima O, Yamauchi Y, Furukawa K, Furukawa K. Wisp2/CCN5 up-regulated in the central nervous system of GM3-only mice facilitates neurite formation in Neuro2a cells via integrin-Akt signaling. Biochemical \& Biophysical Research Communications. 2011; 411:483-489.

30. Tang Z, Yuan XR, Zheng W. Effects of Wisp2 siRNA on proliferation and apoptosis of human glioma U251 cells. Journal of International Neurology \& Neurosurgery. 2013; 40:199-203.

31. Nakanishi C, Nagaya N, Ohnishi S, Yamahara K, Takabatake S, Konno T, Hayashi K, Kawashiri MA, Tsubokawa T, Yamagishi M. Gene and protein expression analysis of mesenchymal stem cells derived from rat adipose tissue and bone marrow. Circulation Journal. 2011; 75:2260-2268.

32. Dmitriev P, Kiseleva E, Kharchenko O, Ivashkin E, Pichugin A, Dessen P, Robert T, Coppée F, Belayew A, Carnac G, Laoudj-Chenivesse D, Lipinski M, Vasiliev A, Vassetzky YS. Dux4 controls migration of mesenchymal stem cells through the Cxcr4-Sdf1 axis. Oncotarget. 2016; 7:65090-65108. https://doi.org/10.18632/oncotarget.11368.

33. Liu N, Patzak A, Zhang J. Cxcr4-overexpressing bone marrow-derived mesenchymal stem cells improve repair of acute kidney injury. American Journal of Physiology Renal Physiology. 2013; 305:1064-1073.

34. Krause DS. Plasticity of marrow-derived stem cells. Gene Therapy. 2002; 9:754-758.

35. Wang Y, Deng Y, Zhou GQ. Sdf-1alpha/Cxcr4-mediated migration of systemically transplanted bone marrow stromal cells towards ischemic brain lesion in a rat model. Brain Research. 2008; 1195:104-112.

36. Yu X, Chen D, Zhang Y, Wu X, Huang Z, Zhou H, Zhang Y, Zhang Z. Overexpression of Cxcr4 in mesenchymal stem cells promotes migration, neuroprotection and angiogenesis in a rat model of stroke. Journal of the Neurological Sciences. 2012; 316:141-149.

37. Fiore EJ, Mazzolini G, Aquino JB. Mesenchymal stem/ stromal cells in liver fibrosis: recent findings, old/new caveats and future perspectives. Stem Cell Reviews and Reports. 2015; 11:586-597.

38. Geiger F, Lorenz H, Xu W, Szalay K, Kasten P, Claes L, Augat $\mathrm{P}$, Richter W. VEGF producing bone marrow stromal cells (BMSC) enhance vascularization and resorption of a natural coral bone substitute. Bone. 2007; 41:516-522.

39. Wang PP, Wang JH, Yan ZP, Hu MY, Lau GK, Fan ST, Luk JM. Expression of hepatocyte-like phenotypes in bone marrow stromal cells after HGF induction. Biochemical \& Biophysical Research Communications. 2004; 320:712-716.

40. Baldwin HS, Shen HM, Yan HC, Delisser HM, Chung A, Mickanin C, Trask T, Kirschbaum NE, Newman PJ, Albelda SM. Platelet endothelial cell adhesion molecule-1 (PECAM-1/CD31): alternatively spliced, functionally distinct isoforms expressed during mammalian cardiovascular development. Development. 1994; 120:2539-2553.

41. Delisser HM, Newman PJ, Albelda SM. Molecular and functional aspects of PECAM-1/CD31. Immunology Today. 1994; 15:490-495.

42. Annabi B, Naud E, Lee YT, Eliopoulos N, Galipeau J. Vascular progenitors derived from murine bone marrow stromal cells are regulated by fibroblast growth factor and are avidly recruited by vascularizing tumors. Journal of Cellular Biochemistry. 2004; 91:1146-1158.

43. Best DH, Coleman WB. Treatment with 2-AAF blocks the small hepatocyte-like progenitor cell response in retrorsineexposed rats. Journal of Hepatology. 2007; 46:1055-1063.

44. Niu Y, Shen B, Cui Y, Chen Y, Wang J, Wang L, Kang Y, Zhao X, Si W, Li W. Generation of gene-modified cynomolgus monkey via Cas9/RNA-mediated gene targeting in one-cell embryos. Cell. 2014; 156:836-843. 